\title{
Speculating Incident Zone System on Local Area Networks
}

\author{
Daichi Hasumi \\ NEC Corporation \\ d-hasumi@ah.jp.nec.com
}

\author{
Shigeyoshi Shima \\ NEC Corporation \\ shima@ap.jp.nec.com
}

\author{
Hiroki Takakura \\ National Institute of Informatics \\ takakura@nii.ac.jp
}

\begin{abstract}
Triage process in the incident handling lacks the ability to assess overall risks to modern cyber attacks. Zoning of local area networks by measuring internal network traffic in response to such risks is important. Therefore, we propose a SPeculating INcident Zone (SPINZ) system for supporting the triage process. The SPINZ analyzes internal network flows and outputs an incident zone, which is composed of devices related to the incident. We evaluate the performance of the SPINZ through simulations using incident flow datasets generated from internal traffic open data and lateral movement traffic. As a result, we confirm that the SPINZ has the capability to detect an incident zone, but removing unrelated devices from an incident zone is an issue to be further investigated.
\end{abstract}

\section{CCS CONCEPTS}

- Security and privacy $\rightarrow$ Malware and its mitigation; • Networks $\rightarrow$ Network measurement;

\section{KEYWORDS}

Cyber Security, Incident Handling, Triage, Anomaly Detection

\section{ACM Reference Format:}

Daichi Hasumi, Shigeyoshi Shima, and Hiroki Takakura. 2018. Speculating Incident Zone System on Local Area Networks. In WTMC'18: ACM SIGCOMM 2018 Workshop on Traffic Measurements for Cybersecurity, August 20, 2018, Budapest, Hungary. ACM, New York, NY, USA, 6 pages. https: //doi.org/10.1145/3229598.3229603

\section{INTRODUCTION}

Information and communications technology (ICT) systems are essential for all organizations to fulfill their missions efficiently. One of their troublesome problems they all face is cyber attacks. Attackers target ICT systems to get secret information, to disrupt operations or to harm the honor of the target. They do this by using malware and taking advantage of software vulnerabilities. Therefore, all organizations need a quick and accurate response to computer security incidents (referred hereafter simply as incident).

In view of this, most organizations monitor security alerts to detect whether or not they are attacked or if an attacker has already invaded their internal network. If a critical incident occurs, in general, a computer security incident response team (CSIRT) member (the responder) isolates suspicious devices from the local network

Permission to make digital or hard copies of all or part of this work for personal or classroom use is granted without fee provided that copies are not made or distributed for profit or commercial advantage and that copies bear this notice and the full citation on the first page. Copyrights for components of this work owned by others than ACM must be honored. Abstracting with credit is permitted. To copy otherwise, or republish, to post on servers or to redistribute to lists, requires prior specific permission and/or a fee. Request permissions from permissions@acm.org.

WTMC'18, August 20, 2018, Budapest, Hungary

(c) 2018 Association for Computing Machinery.

ACM ISBN 978-1-4503-5910-8/18/08 . .\$15.00

https://doi.org/10.1145/3229598.3229603 if it is acceptable from the perspective of their business continuation, acquires log data for a more detail analysis, and remedies the affected devices. These CSIRT activities together are called incident handling [3].

However, the time to detect and contain cyber attacks are too long in comparison with the time the attacker uses to achieve their objective $[17,20]$. To minimize this gap, it is necessary to develop new technologies and methods to support CSIRT operations. Our previous research $[8,9]$ reveals that acquiring $\log$ data from both devices and networks, and identifying affected areas for incidents, are main factors in preventing prompt incident handling. This is caused by the lack of designs and preparedness for the incident handling. If affected areas of the incident is ambiguous, the responder cannot take countermeasures, like isolating devices from the network, limiting network access, etc. Deciding the impact of incidents and determining affected areas have been labeled as part of the triage process in incident handling [2].

Therefore, to address this problem, we propose the SPeculating INcident Zone (SPINZ) system for supporting this triage process. The SPINZ analyzes internal network traffic, and detects the affected area from the incident (incident zone). The incident zone provides useful information about which network addresses are affected, and which devices must be investigated, against cyber attacks with infection expansion. Moreover, we evaluate the performance of the SPINZ through simulations with two representative incident scenarios using internal network traffic.

\section{RELATED WORK}

With increased damage done by advanced persistent threat (APT) and zero-day vulnerability attacks, traffic monitoring and measurements of local networks has become mandatory to grasp cyber attack outlines. Threat detection methods with malware using internal network flows are mainly divided into botnet and lateral movement detection.

Botnet detection methods focus on anomalies in device behaviors [5, 6]. These methods use the correlation between communication trends or patterns of bots. This is because bots belonging to the same bot family have the same, or similar behaviors, according to their architecture.

Detecting lateral movement differs from botnet detection methods. Fawaz et al. [4] proposed a framework to detect lateral movement using distributed data fusion in a large network. Their architecture has three communication graphs at the host, cluster, and global levels. Agents at these three levels cooperate to detect lateral movement activity.

Alternatively, Hasegawa et al.[7] proposed adaptive network control method to minimize damages from APT attacks after detecting an incident. They introduced an infection suspicious level (ISL) for each network segment. Their method isolates network segments 
and devices in the entire network to suppress an attacker's activity, if necessary.

To protect internal networks, a unified system to detect and respond to both botnet and lateral movements is required. However, existing research only considers one threat on either side, or focusing on a detection phase. On the other hand, the SPINZ considers both threats when an incident is detected, and provides support with a triage process to the affected area of the incident.

\section{SPINZ}

To support triage process, we propose the speculating incident zone (SPINZ) system. In this chapter, we describe a concept of the incident zone, the abstract of SPINZ and its calculation algorithm.

\subsection{Incident Zone}

Table 1 shows observable internal network activities in representative malware incident cases [1, 14, 22]. According to Table 1, the most frequent observation activities are communications with $\mathrm{C} 2$ (command \& controls) servers, but the second most frequent observation activities are spreading infections in the local network. This means that it is important to grasp the status of internal infections as much as monitoring communications with the Internet at the gateway, for a detection and response against incidents.

However, in the current triage process, a responder tends to only investigate a device marked with a high importance alert or one that is obviously behaving suspiciously. This investigation method leads to a failure in incident containment when attackers are already under control of multiple devices in the same local network, because they can notice that an attack is detected by receiving signals from infected devices. Therefore, attackers could delete their trails, and evade detection by suppressing their activities.

In this situation, it is necessary to conduct the triage process to determine risks and countermeasures by defining areas where other devices are susceptible to incidents, which we called the incident zone in this paper. Defining the incident zone allows responders to control service availability and business continuity while handling incidents with an accurate risk perception.

\subsection{System Design}

The SPINZ is based on the idea that abnormal communications between the incident device and other devices might be suspicious. The SPINZ is composed of agents and an analyzer. Figure 1 illustrates the overview of the components and process for the SPINZ.

The agent is software installed on a device using the local network. It records network packet headers communicating with other devices on the device where the agent is installed on (payload capture is not necessary). The TCP, UDP, and ICMP are selected as a recorded protocol for the SPINZ. Log data is recorded for each communication session, and each session includes the basic 5-tuple information (source IP, destination IP, source port, destination port, and protocol number) plus the number of packets, bytes per session, session start time and duration. Logged flows are limited to internal communications because the SPINZ focuses on internal malicious activities such as spreading infection and lateral movement. Once a serious incident happens, the agent send logs to the analyzer in accordance with the analyzer's order.
The analyzer sends orders to the agents on target devices to collect the communication log data, and analyzes it to detect the incident zone. There are three basic steps for this analysis.

(Step1) The analyzer defines device roles of the target device and devices communicating with it at regular intervals based on number of transmission bytes. The device role reflects its function on the local network. We adopted two role types: client and server. The client includes the devices an employee commonly uses. In contrast, the server includes devices that provide particular services, for example, file sharing servers, internal web services, authentication, etc. This process focuses on finding cases where roles change on the same device at different intervals. Changing roles on a device between intervals directly suggests a transition in device behaviors. Therefore, the analyzer gives a high score to these cases.

(Step2) Next, the analyzer investigates the target device's traffic $\log$. The purpose of this process is to find and score uncommon communications in accordance with the empirical rules. The result is calculated using the ruleset and the change point detection method.

(Step3) The final process combines the results from Step1 and Step2 to get the activity change score for the target device. The analyzer compares the threshold and the score to determine whether or not the target device belongs in the incident zone. If the target device is put into the incident zone, the analyzer applies Step1-3 calculations to the other devices communicating with the target device. This process loop continues recursively as long as devices for the next calculation exist.

As a result of the above steps, the incident zone is outputted with devices which has a high relevance to the incident. The responder can consider incident risks and respond referring to this incident zone.

\subsection{Scoring Algorithm}

Note that with the detailed calculation method, the analysis period is denoted $A P$, the split period as $S P$, a target device to be calculated the activity change score is $x$, and devices communicating with $x$ in $A P$ are denoted as $y \in \mathbb{Y}_{x}$. Also, $\alpha$ is the initial device on which an incident was detected (the first detected device is added to the incident zone automatically). We also represent a slot sequence as $\left\{w_{1}, w_{2}, \cdots, w_{n}\right\}$ where $n$ is the number of $A P$ divided by $S P$, and $w$ is a time slot window.

3.3.1 Step1: Calculate role-change score. At first, the analyzer defined device's roles in the local network at every window. We adopted two role, Client and Server, because devices in a modern internal network were divided into devices used by common users (Client), and devices that provided particular services (Server). If a Client role device showed infection activities, its role might change to Server role. Therefore, predicting role change was suitable to grasp communication flow changes in the local network. Li et al. [13] studied classification method to separate internal device roles into Client or Server. Their method applied a statistical classifier to long term flow data. However, in the SPINZ, SP is interchangeable for the demand of a responder or a local network environment. Therefore, We used transmission amounts to judge a device's role, because their research claimed that the transmission amount was the most significant variable contributing to a classifier 
Table 1: Observable internal network activities in representative malware incident cases.

\begin{tabular}{lcccccc}
\hline Activity & Stuxnet & Duqu & Flame & Red October & Carbanak & Petya \\
\hline Connect to C2 (Command \& Control) servers & $\checkmark$ & $\checkmark$ & $\checkmark$ & $\checkmark$ & $\checkmark$ & - \\
Spread infections in the internal network & $\checkmark$ & $\checkmark$ & $\checkmark$ & - & - & $\checkmark$ \\
Utilize infected devices for a proxy server & $\checkmark$ & - & - & - & - & - \\
Construct a P2P network between infected devices & $\checkmark$ & $\checkmark$ & - & - & - & - \\
\hline
\end{tabular}
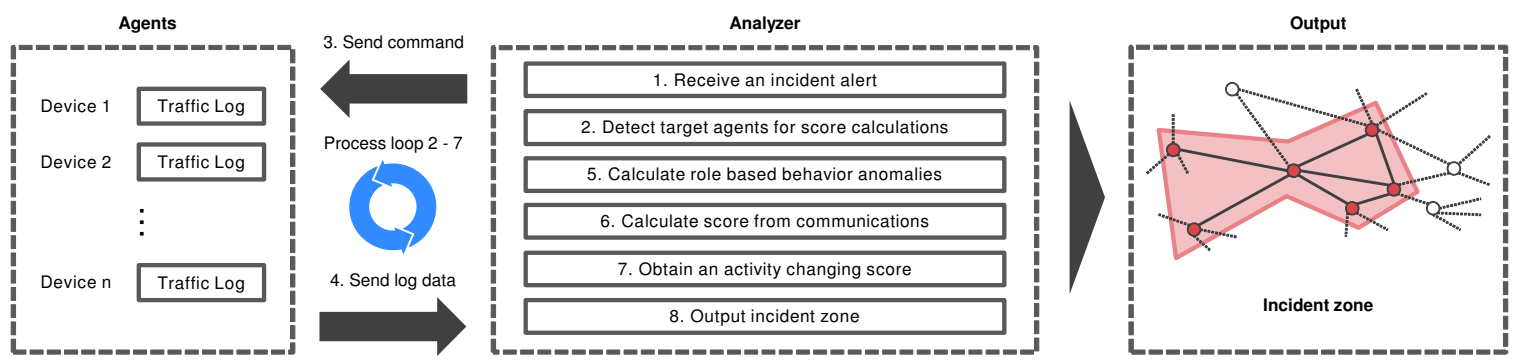

Figure 1: Overview of components and process for the SPINZ. The numbers before each description indicate the SPINZ processing order.

in role detection. The device role for $x$ at $w$ is represented as eq. 1.

$$
\text { Role }_{x, w}= \begin{cases}\text { Client } & \text { if send }(x, w)<\operatorname{receive}(x, w) \\ \text { Server } & \text { otherwise }\end{cases}
$$

$\operatorname{send}(x, w)$ and receive $(x, w)$ represent sending and receiving bytes of $x$ at $w$ from all internal communications. We defined all device roles at every $w$ using eq. 1 .

The analyzer then calculated the role change score $S_{\text {role }}$ of $x$ at every $w . S_{\text {role }}$ is denoted by

$$
S_{\text {role }}(x)=\left\{0, \text { RoleChange }\left(x, w_{2}\right), \cdots, \text { RoleChange }\left(x, w_{n}\right)\right\}
$$

where RoleChange is a function that outputs 0 or 1 based on the previous role of $w$. If a role in a previous and current window were the same, RoleChange outputs 0 , otherwise it outputted 1 . RoleChange $\left(x, w_{1}\right)$ is always 0 , because the first role of $w_{1}$ is regarded as a base role. Note that $S_{\text {role }}$ is an array, and its size is $n$.

3.3.2 Step2: Calculate communication score. Next, the analyzer investigated the communication between $x$ and $y \in \mathbb{Y}_{x}$ at every $w$. Step 2 calculated the communication score $S_{\text {comm }}$ using the role combination rules and change points based on transmission bytes per sessions.

To calculate $S_{\text {comm }}$, we prepared a variation score $\operatorname{var}(x, y, w)$ between the target device $x$ and communicating device $y$ at window w. var was calculated with eq. 3 .

$$
\operatorname{var}(x, y, w)=\left\{\begin{array}{cc}
1 & \text { if } \operatorname{Comb}(x, y, w)=(\text { Client }, \text { Client }) \\
1 & \text { if } \operatorname{Comb}(x, y, w)=(\text { Client }, \text { Server }) \\
& \operatorname{and} c d_{\text {max }}(x, y, w)>T h_{c d} \\
1 & \text { if } \operatorname{Comb}(x, y, w)=(\text { Server }, \text { Client }) \\
& \text { and } c d_{\text {max }}(x, y, w)>T_{c d} \\
1 & \text { if } \operatorname{Comb}(x, y, w)=(\text { Server }, \text { Server }) \\
0 & \text { otherwise }
\end{array}\right.
$$

eq. 3 represents the role combination rules to derive var values. Note that $\operatorname{Comb}(x, y, w)$ is a function that returns a tuple of roles from $x, y$ at $w, c d_{\text {max }}(x, y, w)$ represents the max score calculated by a change point detection method between $x$ and $y$ at $w$, and $T h_{c d}$ is a threshold value to judge variations of communication between devices are enough to suspicious. In this case, role types were limited to two; Therefore, Comb returns one of four types of tuple. Suspicious or unusual communication in the local network was detectable looking at the source device role and the destination one. This suggests that rules for suspicious communication from role combination could be defined, for example, a communication between Client would be suspicious because it did not usually occur. In addition, communications in which large variation was observed were also considered suspicious, even though the role combination was common. Therefore, we incorporated these ideas into the rule set using a change point detection method. We adopted ChangeFinder[19] to detect variations of bytes per session between $x$ and $y$ for each $w$. If the max score of ChangeFinder $c d_{\text {max }}$ exceeds $T h_{c d}$, var is 1 in specific role combinations. For simplicity, ChangeFinder scores are normalized from 0 to 1 .

Subsequently, the analyzer found a maximum var (denoted as $v_{\text {ar }}$ max $)$ in $\mathbb{Y}_{X}$ for each $w$, and constructed an $n$ size array $S_{\text {comm }}$ arranged in order of $w$.

$$
\begin{gathered}
\operatorname{var}_{\text {max }}(x, w)=\max _{y \in \mathbb{Y}_{x}}\{\operatorname{var}(x, y, w) \times I(x, y)\} \\
I(x, y)=-\log _{2} f r e q(x, y) \\
S_{\text {comm }}(x)= \\
\left\{\operatorname{var}_{\text {max }}\left(x, w_{1}\right), \operatorname{var}_{\text {max }}\left(x, w_{2}\right), \cdots, \operatorname{var}_{\text {max }}\left(x, w_{n}\right)\right\}
\end{gathered}
$$

In eq. $5, I(x, y)$ is self-information[18] and $f r e q(x, y)$ represents a frequency of communication of $x$ to $y$ in communications related $x$ during the whole $A P . I(x, y)$ was introduced to take into account rarity of a communication. 
3.3.3 Step3: Merge score. The final step combined $S_{\text {role }}$ and $S_{\text {comm }}$ to calculate the activity change score $S_{a c t} . S_{a c t}$ was calculated by

$$
S_{\text {act }}(x)=\left[m \times S_{\text {role }}(x)+(1-m) \times S_{\text {comm }}(x)\right] \times \sup ^{h o p}(x, \alpha)
$$

where $m$ is a mixture ratio, sup is a suppression factor, $h o p(x, \alpha)$ is a function that returns the number of network hops between $x$ and $\alpha$. This function was introduced for the hypothesis that the incident zone is close to $\alpha$. The range of $m$ and sup are [0,1]. If even one value of $S_{a c t}\left(S_{a c t}\right.$ is a $n$ size array) had a larger value than threshold $T h_{a c t}$, the device $x$ was put into the incident zone, and devices $\mathbb{Y}_{x}$ became the target devices for next calculation. The device whose score had already calculated was not selected as next target again. This calculation loop continued until the next target device no longer existed.

\section{EXPERIMENTAL RESULTS}

For performance evaluation, the SPINZ needs internal network flows including malicious activity. Therefore, We generated an incident flow dataset to evaluate the SPINZ by blending normal internal network flows from an open dataset and typical malicious activity flows from a closed experiment network. In this section, we describe the open dataset we used, incident scenarios, and simulation results of the SPINZ.

\subsection{Dataset}

As a normal internal network flow, we used an open dataset from Los Alamos National Laboratory (LANL) [11, 12]. The LANL dataset contained 129,977,412 session flows over 29 days, and recorded nine items per line: [start time, duration, source IP, source port, destination $I P$, destination port, protocol number, number of packets, transmission bytes]. These flows were only for internal communications in the LANL network. Figure 2 shows the part of logs in the LANL dataset. The LANL dataset contains attributes separated by commas in each row. A row represents a separate communication session.

For the evaluation, we removed traffic flows related to core service servers from the LANL dataset, because core service servers such as authentication servers and mail servers were already generally monitored. Therefore, they were outside the scope of the SPINZ. Thus, we made a discrimination based on the number of communications with other IPs (degrees) for each IP in the LANL dataset. Figure 3 shows a degree distribution plot when a graph is constructed with each IP as a node and each communication between different IPs as an edge in LANL dataset. The $x$-axis represents the number of degree of each IP. Also, the y-axis represents the fraction of nodes with the corresponding degrees. From Figure 3, we found almost IP has under 100 degrees and few IPs has extremely large degrees. This means that core service servers could be divided from all IPs by the number of degrees. When arranging IP in degree order, we defined that top $0.5 \%$ IPs which had over 204 degrees as core service servers, under 95\% IPs which had under 24 degrees as common devices, and other $4.5 \%$ IPs as common servers. Finally, we removed flows with core service serves from the LANL dataset.

Additionally, we also prepared typical lateral movement flows from the closed experimental network. We observed scan and file

\begin{tabular}{|c|c|c|c|c|c|c|c|c|}
\hline 476233 & 0 , & C5721, & 445 & $\begin{array}{l}\vdots \\
\text { C18654, }\end{array}$ & N13539, & 6, & 1, & 168 \\
\hline 476233, & 0 , & C5721, & 445 & C24495, & N5954, & 6 & 1 , & 91 \\
\hline 476233, & 0, & C5736, & 445 & C19684, & N1948, & 6 & 1 , & 91 \\
\hline 476233, & 0, & C585, & 139 & C9610, & N3746, & 6 & 1, & 46 \\
\hline 476233, & 0, & C586, & 389 & C19191, & N30818, & 17 & 1, & 193 \\
\hline 476233, & 0, & C586, & 389, & C958, & N30817, & 17 & 1 , & 193 \\
\hline 476233, & 0 , & C706, & 80 & C19662, & N26051, & 6 , & 6 , & 4220 \\
\hline 476233, & 0, & C706, & 80 & C3785, & $\mathrm{N} 16685$, & 6 , & 5, & 557 \\
\hline
\end{tabular}

Figure 2: Part of logs in the LANL dataset.

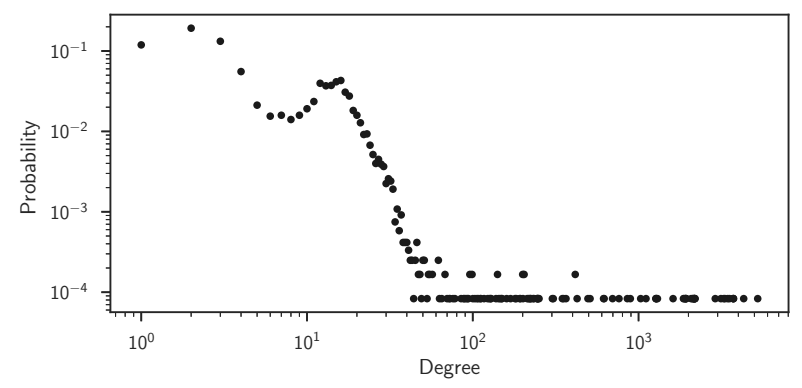

Figure 3: Degree distribution in the LANL dataset.

sharing flows using net command and PsExec [15], between two machines installed with Windows 7. Detailed commands we inputed on were described below.

(1) net view [Target]

(2) psexec [Target] -u [user name] -p [password] xcopy "[Source $\backslash$ share $\backslash \backslash$ malware" "c: $\backslash \backslash "$

[Source] was an IP address trying to infect to other devices and [Target] was an IP address infected from the [Source]. [user name] and [password] were [Target]'s authentication information. In addition, Emdivi RAT T17.08.34 which is malware used for actual APT attacks was chosen as a file to be transmitted. A net command and PsExec are general tools found in APT incident cases [10]. Therefore, we regarded these flows as typical malicious activity flows. These session flows were recorded in the same format as the LANL dataset.

We then mixed malicious activity flows into the LANL dataset. Mixed malicious flows could not be distinguished from normal flows. What time and how much malicious flows are added to the LANL dataset depended on the incident scenario which be explained to the next section.

\subsection{Incident Scenario}

To generate the incident flow dataset, two parameters, such as an infection count $(I C)$ and an infection frequency $(I F)$, were required based on incident cases. $I C$ represented a total infected count in the incident scenario, and $I F$ represented an interval between infected flows. These two parameters had a relation of $I F=I C / T_{a t k}\left(T_{a t k}\right.$ represented the total attack period, we assumed that all attacks were performed within the duration of $A P$ in this experiment).

In this paper, we used two incident scenarios: shallow infection and deep infection. 
Shallow infection was a scenario assuming an infection method of malwares, such as traditional bots or ransomwares, which infections spread from an infected device to many local devices in a short time. In this scenario, we set $I C$ as 37 (from the National Audit Office report [16]) and IF to one second.

Also, deep infection was a scenario assuming an infection method of a typical APT case, where an infection spread from an infected device to a specific target device in accordance with the purpose of the attacker over a long time. In this scenario, we set $I C$ as five and $I F$ to 24 hours. This means the attacker infected another device once a day, and the attacker repeated this five times. This behavior simulated an attacker's "deep" movement in the local network, and is difficult to detect using statistical anomaly detection methods.

Figure 4 is an example visualization of two incident scenarios. The black node shows the initially infected device, and it spreads to other devices for each infection frequency.

\subsection{Simulation}

Finally, we evaluated the SPINZ through simulations using the incident flow dataset with two incident scenarios. The purpose of evaluation was to confirm reliability of outputted incident zones. Therefore, we adopted recall and precision as performance measurements. Both definitions are described in eq. 8 and eq. 9.

$$
\begin{gathered}
\text { Recall }=\frac{T P}{T P+F N} \\
\text { Precision }=\frac{T P}{T P+F P}
\end{gathered}
$$

In both of eq. 8 and eq. $9, T P$ is a true positive rate, $F P$ is a false positive rate, and $F N$ is a false negative rate from the result of the SPINZ and incident scenarios. Recall value indicated how much the incident zone contained actual related devices, and precision value indicated how close the size of the incident zone was to actual one. The initially infected device and attacked devices were randomly selected in each simulation execution. In this simulation, the initially infected device was limited to a common device, because attackers generally infect common devices via email or those with critical vulnerabilities in modern cyber attacks [21]. Table 2 shows parameters for the SPINZ. we set the same parameters for both incident scenarios, but $I C$ and $I F$ were dependent on each scenario. Note that we used first 14 day sessions from original one in accordance with AP. Although the size of the final dataset inputed to the SPINZ was smaller than the whole LANL dataset, 14 day sessions contained 11,504 IPs, and 114,560,974 flows. Therefore, we judged that these sessions were sufficient as a dataset of the large local area network.

The ChangeFinder parameter was the same as the original paper $(k=2, T=5)$, but the discounting parameter $r$ was 0.1 as shown in Table 2. We also changed $S P$ to one hour, three hours, six hours, 12 hours, 24 hours to evaluate performance change. In each $S P$ parameter, simulations were performed 20 times and the average values ware plotted to Figure 5.

Figure 5 shows the simulation results for shallow infection (a) and deep infection (b). For both results, the first y-axis shows average recall, the second $\mathrm{y}$-axis shows average precision. Also, the $\mathrm{x}$-axis represents $S P$. In the result, the average recall was quite high, and a large change was not observed in each $S P$ graph. However, the

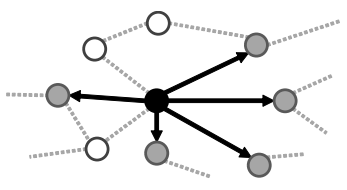

(a) Shallow Infection scenario $(I C=5)$

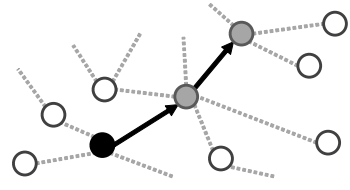

(b) Deep Infection scenario $(I C=3)$
Figure 4: Example visualization of two incident scenarios. black node represents an incident device, and gray nodes represent attacked devices.

Table 2: Parameters for simulation.

\begin{tabular}{lc|lc}
\hline Parameter & Value & Parameter & Value \\
\hline AP & 14 days & sup & 0.95 \\
$T h_{c d}$ & 0.75 & Attack Start Time & 7.5 days \\
$T h_{\text {act }}$ & 0.4 & $r$ (for ChangeFinder) & 0.1 \\
$m$ & 0.5 & & \\
\hline
\end{tabular}

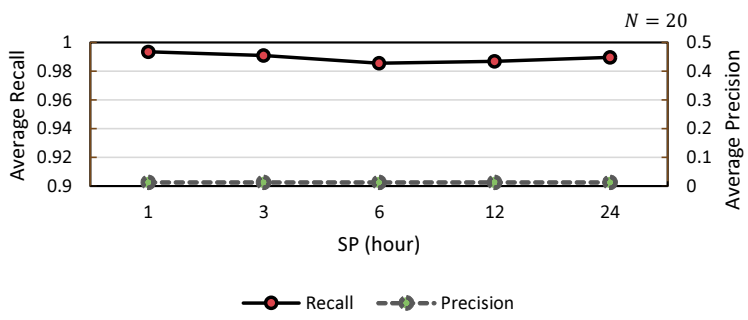

(a) Shallow infection (simulation time $N=20$ ).

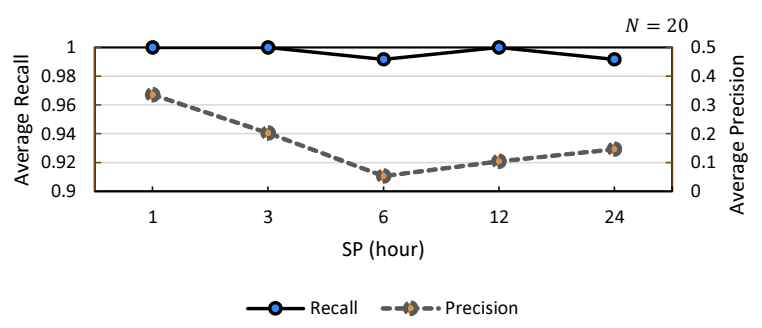

(b) Deep infection (simulation time $N=20$ ).

Figure 5: Simulation results.

average precision overall was low. All average recalls and precisions through $S P$ s were $(0.99,0.01)$ in Figure $5(\mathrm{a})$, and $(0.99,0.17)$ in Figure 5 (b)

\section{DISCUSSION}

The SPINZ showed good recall values for both incident scenarios. This means that the SPINZ outputted incident zones that included almost devices related to the incident by analyzing the internal network flows without any specific knowledge about the incident 
and cyber attacks. The SPINZ would be great help to responders when they make initial decisions about an incident in the triage process, because missing devices related to the incident leads to failure in the incident handling.

However, precision values were remarkably low in the shallow infection. A low precision value indicated that the SPINZ outputted an inaccurate incident zone with unrelated devices. This was caused by the SPINZ outputs for an incident zone only considering the role of devices, role combination rules, and communication fluctuation. Interestingly, the precision values were better in the deep infection. This was because the number of communication devices in the initial incident device was few, and the expansion of the incident zone was suppressed. In general, the internal network flow fluctuates daily, because when employees process different tasks, they generate different communication patterns. We considered that the easiest solution to improve precision was to limit the number of flows inputted to SPINZ. For example, it was conceivable to input only the flow whose destination port was 445 into the SPINZ. Considering the actual operation, A size of the incident zone could be smaller as the responder progresses the investigation, because the responder could mark devices investigated detail as related devices or not. Thus, the SPINZ reflects these results to an incident zone.

\section{CONCLUSION AND FUTURE WORK}

Triage is significant process for successful incident handling. However, the triage process lacks an ability to recognize risk in other devices and the local network against cyber attacks. We considered zoning the local area network with risk recognition by measuring internal network traffic as important for incident handling operations. Therefore, we proposed a SPeculating INcident Zone (SPINZ) system. The SPINZ analyzes internal network flows and outputs an incident zone that includes devices related to the incident. By defining high risk devices as part of an incident zone, a responder can appropriately recognize a risk and prompt an initial countermeasure to the incident.

We also evaluated the performance of the SPINZ through simulations with a bot and lateral movement infection scenarios. we used an incident flow dataset generated from internal traffic dataset and malicious activity traffic. As a result, the SPINZ could output incident zones that included devices related to the incident. However, we also found that a large number of unrelated devices were included in the incident zone.

For future work, we continue to work on improving the precision value. The most important issue is a large false-positive rate proportional to the number of target devices. We consider there is a possibility of restricting the number of calculating devices according to network characteristics and topology. Moreover, we are going to develop parameter adaption methods, and evaluate robustness when real network flows applied.

\section{ACKNOWLEDGMENT}

The authors would like to thank anonymous reviewers and our colleagues for their insightful comments and suggestions improving the manuscript significantly.

\section{REFERENCES}

[1] WJB Beukema. 2016. Enhancing Network Intrusion Detection through Host Clustering. Master's thesis. University of Twente, Drienerlolaan, The Netherlands.

[2] Moira West Brown, Don Stikvoort, Klaus-Peter Kossakowski, Georgia Killcrece, Robin Ruefle, and Mark Zajicek. 2013. Handbook for computer security incident response teams (CSIRTs). Technical Report CMU/SEI-2003-HB-002 , Carnegie Mellon SEI. (2013).

[3] Paul Cichonski, Tom Millar, Tim Grance, and Karen Scarfone. 2012. Computer security incident handling guide. NIST Special Publication 800, 61 (2012).

[4] Ahmed Fawaz, Atul Bohara, Carmen Cheh, and William H. Sanders. 2016. Lateral Movement Detection Using Distributed Data Fusion. In 2016 IEEE 35th Symposium on Reliable Distributed Systems (SRDS'16). 21-30.

[5] Guofei Gu, Phillip Porras, Vinod Yegneswaran, and Martin Fong. 2007. BotHunter: Detecting Malware Infection Through IDS-Driven Dialog Correlation. In 16th USENIX Security Symposium (USENIX Security 07). USENIX Association.

[6] Guofei Gu, Junjie Zhang, and Wenke Lee. 2008. BotSniffer: Detecting Botnet Command and Control Channels in Network Traffic. In Proceedings of the 15th Annual Network and Distributed System Security Symposium (NDSS'08).

[7] Hirokazu Hasegawa, Yukiko Yamaguchi, Hajime Shimada, and Hiroki Takakura. 2016. An incident response support system based on seriousness of infection. In 2016 International Conference on Information Networking (ICOIN). IEEE, 69-74.

[8] Daichi Hasumi, Shigeyoshi Shima, and Takahiro Kakumaru. 2016. Issue analysis toward forensics gathering infrastructure that supports the more efficient incident handling. The Special Interest Group Technical Reports of IPSf 2016-SPT-17, 7, $1-6$.

[9] Daichi Hasumi, Shigeyoshi Shima, and Hiroki Takakura. 2017. Proposal of the triage supporting system for the more efficient incident handling. In 2017 Symposium on Cryptography and Information Security (SCIS).

[10] JPCERT/CC 2017. Detecting Lateral Movement through Tracking Event Logs. (2017). Retrieved Jun 7, 2018 from https://www.jpcert.or.jp/english/pub/sr/ir_ research.html

[11] Alexander D. Kent. 2015. Comprehensive, Multi-Source Cyber-Security Events. Los Alamos National Laboratory. (2015). https://doi.org/10.17021/1179829

[12] Alexander D. Kent. 2015. Cybersecurity Data Sources for Dynamic Network Research. In Dynamic Networks in Cybersecurity. Imperial College Press.

[13] Bingdong Li, Mehmet Hadi Gunes, George Bebis, and Jeff Springer. 2013. A supervised machine learning approach to classify host roles on line using sFlow. In Proceedings of the first edition workshop on High performance and programmable networking (HPPN 2013). ACM, 53-60.

[14] Mark Simos 2018. Overview of Petya, a rapid cyberattack. (2018). Retrieved Jun 7, 2018 from https://cloudblogs.microsoft.com/microsoftsecure/2018/02/05/ overview-of-petya-a-rapid-cyberattack/

[15] Microsoft 2016. Microsoft TechNet Windows Sysinternals PsExec. (2016). Retrieved Jun 7, 2018 from https://docs.microsoft.com/en-us/sysinternals/ downloads/psexec

[16] National Audit Offece 2017. Investigation: WannaCry cyber attack and the NHS. (2017). Retrieved Jun 7, 2018 from https://www.nao.org.uk/report/ investigation-wannacry-cyber-attack-and-the-nhs/

[17] Ponemon Institute 2017. 2017 Cost of Data Breach Study Global Overview. (2017). Retrieved Jun 7, 2018 from https://www-01.ibm.com/common/ssi/cgi-bin/ ssialias?htmlfid=SEL03130WWEN

[18] John G. Proakis and Masoud Salehi. 2002. Communication Systems Engineering (2 ed.). Prentice Hall Inc., Upper Saddle River, NJ, USA

[19] Jun-ichi Takeuchi and Kenji Yamanishi. 2006. A unifying framework for detecting outliers and change points from time series. IEEE Transactions on Knowledge and Data Engineering 18, 4 (2006), 482-492.

[20] Verizon 2016. 2016 Data Breach Investigations Report. (2016). Retrieved Jun 7, 2018 from https://www.verizonenterprise.com/resources/reports/rp_DBIR_2016_ Report_en_xg.pdf

[21] Verizon 2018. 2018 Data Breach Investigations Report. (2018). Retrieved Jun 7, 2018 from https://www.verizonenterprise.com/resources/reports/rp_DBIR_2018_ Report_en_xg.pdf

[22] Nikos Virvilis and Dimitris Gritzalis. 2013. The Big Four - What We Did Wrong in Advanced Persistent Threat Detection? In 2013 International Conference on Availability, Reliability and Security (ARES 2013). 248-254. 